\title{
The Molecular Universe
}

\author{
A. G. G. M. Tielens ${ }^{1}$ \\ ${ }^{1}$ Leiden Observatory, Leiden University, \\ PO Box 9513, NL-2300RA, Leiden, the Netherlands \\ email: tielens@strw.leidenuniv.nl
}

\begin{abstract}
Over the last 20 years, we have discovered that we live in a molecular Universe: A Universe with a rich and varied organic inventory; A Universe where molecules are abundant and widespread; A Universe where molecules play a central role in key processes that dominate the structure and evolution of galaxies; A Universe where molecules provide convenient thermometers and barometers to probe local physical conditions; A Universe where molecules can work together to form such complex species as you and me. Understanding the origin and evolution of interstellar and circumstellar molecules is thus key to understanding the Universe around us and our place in it and has become a fundamental goal of modern astrophysics. This review focuses on the organic inventory and the chemical processes that may play a role in stablishing molecular complexity in regions of planet formation.
\end{abstract}

\section{Introduction}

The field of astrochemistry is heavily driven by new observational tools that have become available over the last 20 years; in particular, space based missions that have opened up the IR and submillimeter window at an ever accelerating pace. Our progress in understanding the Molecular Universe is greatly aided by close collaborations between astronomers, molecular physicists, astrochemists, spectroscopists, and physical chemists who work together in loosely organized networks. In this review, I will sketch the progress that we have made over the last 20 years and outline some of the challenges that are facing us. The focus will be on understanding the unique and complex organic inventory of regions of star and planet formation that may well represent the prebiotic roots to life. Astrochemistry is much broader that this and key questions such as "What is the role of molecules in the evolution of the Universe ?" and "How can we use molecules to study the Universe ?" are by necessity not addressed here.

Over the last twenty years, we have seen a major shift in our thinking on life in the Universe. First, ground-based Doppler and transient studies have lead to the discovery of $\sim 1700$ exoplanets (and counting). Sixty of these resemble the Earth and at the end of the Kepler mission, we can expect to know some 200 terrestrial planets. Of the 1700 suspected planets, some 50 are in the habitable zone of their star and 5 of those are Earth-like. This census is very much incomplete and we are only starting to address the general architecture of planetary systems and how this compares to the Solar system, but already it is clear that planetary systems are common. Second, in biology, it has become clear that life can adapt itself to very extreme conditions. Life can thrive at very low and at very high temperatures, under very alkaline, very acidic, and very saline conditions, in very arid regions (and wet regions, duh): Extremophiles are everywhere and consequently the habitable zone of planetary systems may be quite large. Liquid water often taken as the defining factor of the habitable zone - may also be more widespread than previously thought and moons such as Europa may have an "internal" ocean in which (primitive) life can be hypothesized. In the Solar system, the presence of liquid 
water has been established - albeit in the past - on Mars and some meteoritic parent bodies. Hence, conditions suitable for life may be widespread in the Universe. Third, the oldest preserved cells date back some 2.5 billion years and a number of possible, or perhaps even likely, indicators for the existence of microbial fossils have been found in rocks that date back to some 3.5 billion years ago. That latter date is very close to the age of the late heavy bombardment when the interaction between Jupiter and Saturn reordered the Solar system. The impacts associated with this bombardment would have sterilized the Earth (over and over again) and so this timeline would leave only $\sim 200$ Myr for the emergence of the first cells on Earth. In all, these new developments have completed the Copernican/Darwinian revolution and the consensus is now that life is widespread in the Universe.

With this shift in paradigm, astrochemistry and its relationship to the prebiotic roots of life have emerged as key areas of research in astronomy. This can be taken at two levels: The first question is: "What are the main reservoirs of carbon in regions of planet formation ?" Or alternatively, "How and in what form was carbon delivered to planets in the habitable zone ?" Obviously, if most of the carbon is in the form of very volatile species (e.g., $\mathrm{CO}, \mathrm{CH}_{4}$ ), then delivery has to be by cometary or icy-asteroidal bodies presumably during a "late heavy bombardment" phase in the assemblage of planetary systems. Alternatively, most of the carbon may be locked up in macromolecular moieties and incorporation into planets may then occur "in-situ" during the growth of planetary embryo's. These different reservoirs result thus in a very different history and very different carbon content of newly formed planets. The second and deeper level that we can examine in the relationship between astrochemistry and the origin of life is: "Does astrochemistry "prime" regions of planet formation for the emergence of life ?" Or phrased alternatively: "Does astrochemistry jump start life's chemistry by delivering the right molecular building blocks (e.g., amino acids) that can be readily assembled in any Darwinian pond ?" In this scenario, astrochemistry takes the place of the Urey-Miller experiment $\dagger$, which actually may not have been very relevant for conditions on the early Earth. We can identify a number of scenarios differing in the importance of astrochemistry for the origin of life: Did the Universe "deliver" 1) carbon which got converted into life's building blocks on the early Earth (Urey-Miller); 2) a primed organic mix (e.g., $\mathrm{H}_{2} \mathrm{CO}, \mathrm{HCN}$ ) which then were converted into e.g., amino acids on the early Earth; 3) specific molecules with well defined biological functions (e.g., amino acids, lipids) which were immediately assembled into cells after being dropped into Darwin's little pond.

The focus of this review is on the main reservoirs of molecular carbon in regions of star and planet formation - CO and PAHs - and the astrochemical processes that "release" this carbon and make it available for the formation of species that might be of interest from a prebiotic point of view. Much carbon may be in the form of soot particles, solid kerogen-like macromolecular structures or otherwise frozen out of active chemistry and these reservoirs are of no concern to this review except to note that if these are the reservoirs life grew from, astrochemistry has little prebiotic relevance.

\section{Overview}

Much of the carbon in regions of star and planet formation is locked up in very stable species that do not readily partake in chemical reactions. The story of interstellar organic

$\dagger$ In the Urey-Miller experiment, the discharge converts simple molecular feedstock in formaldehyde and cyanide that are subsequently converted into amino acids through Strecker synthesis in water. 


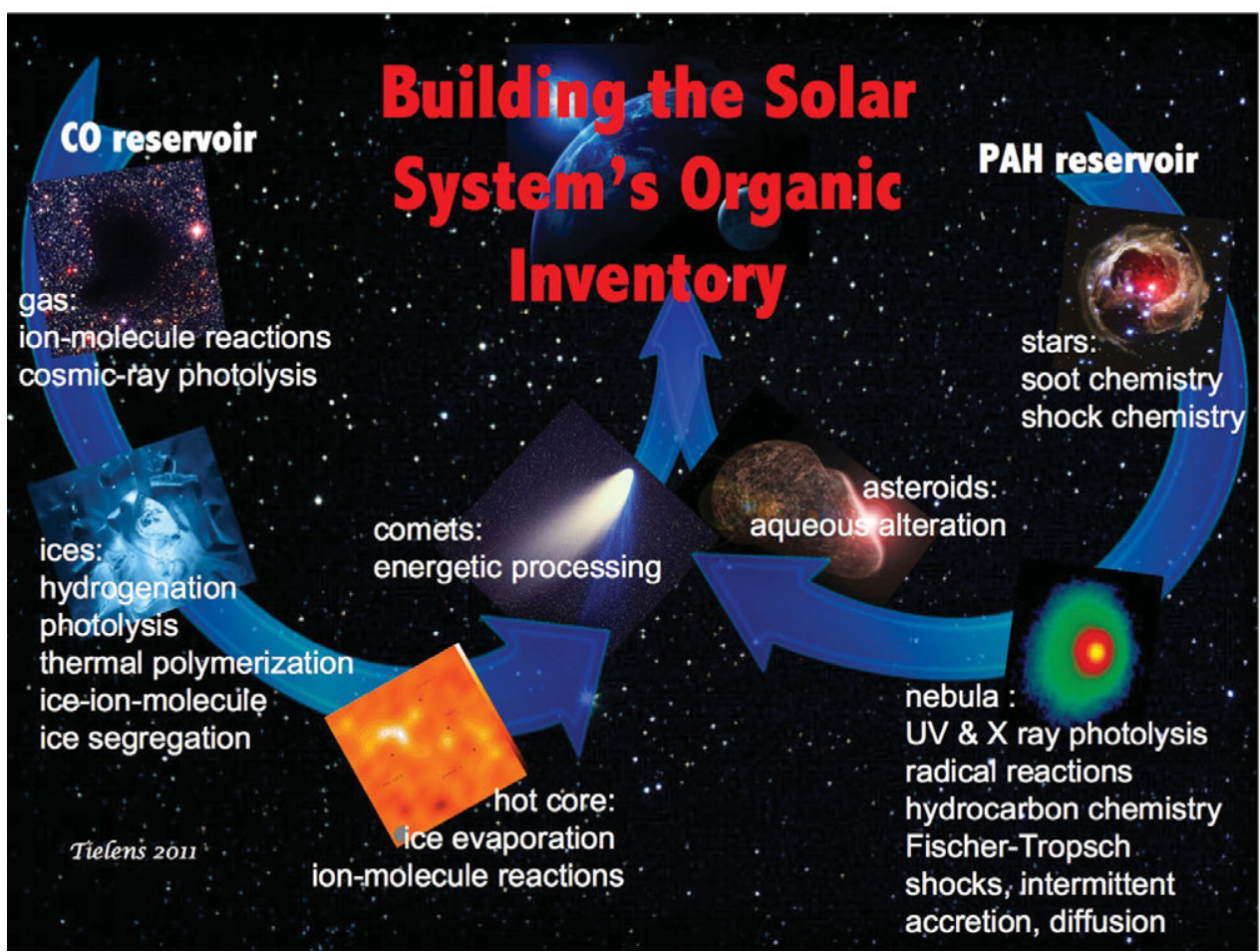

Figure 1. The organic inventory of the Solar System derives from a vast array of processes acting in a wide range of environments. Globally, two independent routes can be recognized. The first one builds up complex species from small radicals and starts with $\mathrm{CO}$ in dark clouds. Ion-molecule reactions, grain surface chemistry, and photoprocessing converts the main gaseous reservoir, CO, into complex species. The other route breaks down very complex species (e.g., PAHs) injected into the interstellar medium by stars into smaller and smaller species. Eventually, the species produced by either of these two chemical routes can become part of planetesimals and cometesimals in a protoplanetary disk environment which deliver this organic inventory to the nascent planets in the habitable zone.

chemistry is therefore by necessity a story that starts with breaking the carbon out of these species. The two main molecular reservoirs are CO and Polycyclic Aromatic Hydrocarbon (PAH) molecules. Figure 1 illustrates relevant chemical routes. In dark clouds, ion molecule chemistry converts gaseous carbon into predominantly carbon monoxide. After accretion onto grains, hydrogenation reactions convert this molecule efficiently into formaldehyde and methanol as well as traces of other species in an icy grain mantle. Energetic processing of these ices by UV photons and cosmic rays can convert these molecules into more complex species, while heating by the protostar can initiate thermal polymerization reactions. The newly formed protostar will sublime these ices and fast ions in shocks can sputter some molecules into the gas phase. Subsequent ion-neutral and neutral-neutral reactions in the warm dense gas of the hot core surrounding the protostar may convert, in particular, methanol into more complex species such as dimethyl ether and methyl formate. These can then accrete again into ices and other planetesimals.

The other route starts with the formation of PAHs and other large, complex molecules in stellar ejecta through chemical processes akin to soot chemistry. The injected PAH family is further processed in the interstellar medium by energetic photons and particles, weeding out the less stable members. Further processing can occur in the photosphere of the protoplanetary disk by stellar UV and X-ray photons. Furthermore, in the inner, hot 


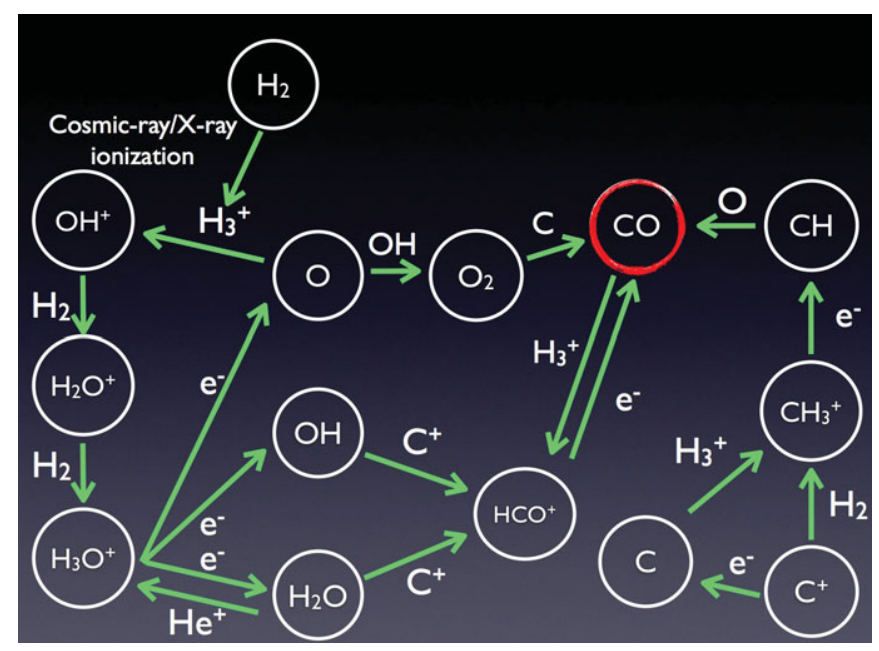

Figure 2. First chemical steps in ion-molecule route convert $\mathrm{C}$ predominantly into $\mathrm{CO}$ in dark clouds (Herbst \& Klemperer 1973; Watson 1973). Figure adapted from Bergin (2011).

and dense (mid-plane) regions, $\mathrm{H}$ and $\mathrm{OH}$ radicals will breakdown these complex species into acetylene, methane and carbon monoxide and carbon dioxide. Turbulence will diffuse these species and their daughter products - formed through neutral-neutral reactions throughout the protoplanetary disk. Catalytic Fischer-Tropsch reactions on iron grains may also be of importance. Eventually, the species produced in these two chemical routes can become part of planetesimals and cometesimals where further processing may occur before this organic inventory is delivered to nascent planets.

\section{The CO reservoir}

\subsection{Ion-molecule chemistry in dark clouds}

Gas phase chemistry in dark clouds is predominantly ion-molecule chemistry where cosmic ray ionization of $\mathrm{H}_{2}$ forms $\mathrm{H}_{2}^{+}$which reacts on with $\mathrm{H}_{2}$ to form protonated molecular hydrogen, $\mathrm{H}_{3}^{+}$(fig. 2). The proton is then efficiently handed over to other atoms and molecules until recombination occurs to a small molecule or radical. The upshot of it all is that ion-molecule chemistry converts all elemental carbon into CO on a very short timescale; e.g., essentially for every $\mathrm{C}$-atom, one $\mathrm{H}_{2}$ has to be ionized $\left(\tau_{\text {chem }} \simeq \mathcal{A}_{\mathcal{C}} / \zeta_{C R} \simeq 2 \times 10^{6}\right.$ yr with $\mathcal{A}_{\mathcal{C}}$ the carbon abundance and $\zeta_{C R}$ the cosmic ray ionization rate. Along the way, the small hydrocarbon radicals may (temporarily) form unsaturated hydrocarbon species such as acetylene and acetylenic derivatives but eventually, and largely, carbon is burned to carbon monoxide.

Molecular complexity is not one of the defining characteristics of ion-molecule chemistry. Observed abundances of even such simple (saturated) species such as $\mathrm{CH}_{3} \mathrm{OH}$ and $\mathrm{NH}_{3}$ cannot be explained by gas phase routes. Also, while acetylenic derivatives such as the cyanopolyynes $\left(\mathrm{eg} ., \mathrm{HC}_{3} \mathrm{~N}\right)$ are a natural exponent of ion-molecule chemistry, their absolute abundance can only be explained well if there is an efficient way to break the $\mathrm{C}$ out of CO. One mechanism to do so is through cosmic-ray-produced UV photons, but the effect is limited: Few of these photons dissociate $\mathrm{CO}$ and the $\mathrm{C} / \mathrm{CO}$ abundance produced this way is only $\simeq 6 \times 10^{-3}$. Reactions with $\mathrm{H}_{3}^{+}$and carbon insertion reactions form then simple hydrocarbons such as $\mathrm{CH}_{4}$ and $\mathrm{C}_{2} \mathrm{H}_{2}$ which can react on to other hydrocarbon species including the cyanopolyynes. Much higher abundances of acetylenic species can 
be produced during early times before all the carbon has been locked up in CO but it is hard to imagine that molecular clouds are typically that young. There are other ways that $\mathrm{C}$ can be broken out of $\mathrm{CO}$ (eg., shocks) but then shocks have to efficiently process clouds on a large scale. I should point out here that some key aspects are not understood; e.g., the observed variation in the abundance of these acetylenic derivatives and what makes some clouds carbon-chain-rich (Hirota et al. 2009) and that may hold important clues.

In summary, with qualifications, gas-phase models of interstellar clouds have been very successful. Among the key successes are explanations for the high abundance of CO, the presence of ions such as $\mathrm{H}_{3}^{+}$and $\mathrm{HCO}^{+}$, the presence of radicals such as $\mathrm{OH}$ and $\mathrm{CH}$, the presence of isomers such as $\mathrm{HNC} / \mathrm{HCN}$, the rich array of acetylenic derivatives present, including the cyano-polyynes, and the high observed deuteration levels in species such as $\mathrm{DCO}^{+}$. Qualitatively, the agreement between gas-phase models and the observations is very impressive. Quantitatively, however, the agreement is wanting. The most extensive reaction networks contain some 4000 reactions among 400 species, and reach an order of magnitude agreement with observed abundances for some $80 \%$ of the species. Partly, this is because our chemical knowledge is imperfect: less than $20 \%$ of the reactions included have actually been studied in the laboratory. The remainder are educated guesses. It is not even known whether all the important chemical routes are included and whether perhaps some routes have been included that are inhibited at low temperature. This is one area of research where we should focus our attention through close collaborations between astrochemical modelers and chemists (Wakelam et al. 2010).

For the story of this review, the bottom line is that in terms of molecular complexity gas phase ion-molecule chemistry is in essence a dead-end, locking the carbon away in a very unreactive species that, from a prebiotic point of view, is unimportant. And this view is confirmed by observations with simple-organics/CO ratios of $\simeq 10^{-3}$ and complex-organics/CO ratios of $\simeq 10^{-5}$.

\subsection{Grain surface chemistry and interstellar ices}

Grain surface chemistry involves predominantly hydrogenation and oxidation reactions and leads to the formation of simple saturated species; e.g., $\mathrm{H}_{2} \mathrm{O}, \mathrm{CO}, \mathrm{CO}_{2}, \mathrm{CH}_{3} \mathrm{OH}$, $\mathrm{H}_{2} \mathrm{CO}, \mathrm{CH}_{4}$, and $\mathrm{NH}_{3}$. Focusing here on hydrogenation, atomic hydrogen is mobile, has a long residence time and because it is so light can tunnel through appreciable activation barriers (Tielens \& Hagen 1982). Hence, H will "collide" often with coreactants on a grain surface and is very reactive. Reactions of $\mathrm{H}$ with $\mathrm{CO}$ and $\mathrm{H}_{2} \mathrm{CO}$ are particularly interesting here, since this transforms $\mathrm{CO}$ first into formaldehyde and then methanol (Fig. 3). Over the past 10 years, these theoretical ideas have been extensively investigated in the laboratory and the viability of this activation route of $\mathrm{CO}$ is now well established (Hiraoka et al. 1998; Hidaka et al. 2004; Fuchs et al. 2009; Ioppolo et al. 2008). This route can also lead to more complex species because the radical intermediaries, HCO and $\mathrm{CH}_{3} \mathrm{O}$, may react with accreted $\mathrm{C}$ (and $\mathrm{O}$ ) atoms rather than $\mathrm{H}$ atoms (Fig. 3). The mobility of $\mathrm{C}$ and $\mathrm{O}$ atoms on a grain surface are not well known and matrix studies yield puzzling results (Tielens \& Allamandola 2011), but if these atoms are mobile a rich chemistry can be initiated. Possible routes towards larger organic compounds are indicated in Figure 3. However, unlike the initial steps towards $\mathrm{H}_{2} \mathrm{CO}$ and $\mathrm{CH}_{3} \mathrm{OH}$, these routes towards further chemical complexity have not been tested experimentally and isomerization at intermediate steps may conceivably lead to other products instead. I do want to point out that, while the precise chemical routes are not yet well characterized, they are expected to be chemical specific and isomers can be expected to have very different abundances. 
A. G. G. M. Tielens

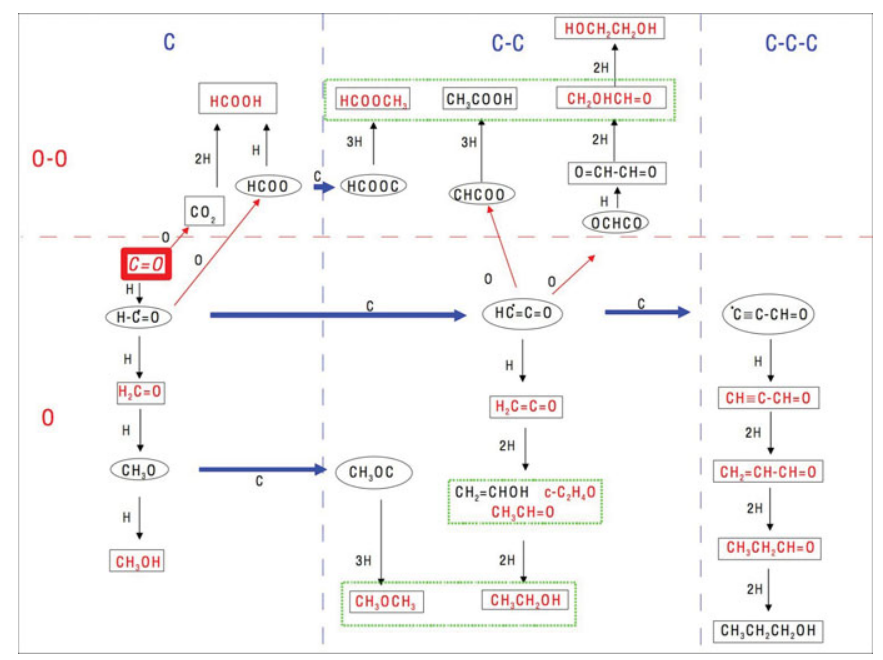

Figure 3. $\mathrm{CO}$ accreted on a grain surface is activated through reaction with $\mathrm{H}$. Eventually these H-reactions will form $\mathrm{H}_{2} \mathrm{CO}$ and $\mathrm{CH}_{3} \mathrm{OH}$ (black arrows). Intermediate radicals such as $\mathrm{HCO}$ and $\mathrm{CH}_{3} \mathrm{O}$ can react with other atoms leading to larger organic molecules (red and blue arrows) and possible routes towards, among others, $\mathrm{HCOOH}, \mathrm{CH}_{3} \mathrm{CH}_{2} \mathrm{OH}$, and $\mathrm{CH}_{3} \mathrm{OCH}_{3}$ are indicated. Figure taken from Requena-Torres et al. (2008), adapted from Tielens \& Hagen (1982); Charnley \& Rodgers (2009).

Grain surface chemistry models have been very successful in qualitatively explaining the molecular inventory of interstellar ices: the high degree of saturation, the importance of methanol, and the presence of hydrogenated and oxidized species. In addition, the high deuteration of formaldehyde and methanol, including the presence of doubly and triply deuterated species, in hot cores is often - and probably rightfully so - cited in support of the importance of grain surface chemistry. Quantitatively, grain surface chemistry models suffer from a lack of detailed reaction rates and branching ratios but we are now reaching the point where quantitative laboratory studies become feasible and, by the next astrochemistry symposium, we should strive to have reliable, quantitative models in hand.

From the point of view of this review, grain surface chemistry is an efficient way of "activating" the $\mathrm{C}$ in $\mathrm{CO}$. Observationally, the ratio of $\mathrm{CH}_{3} \mathrm{OH} / \mathrm{CO}$ can reach values as high as 1 in interstellar ices. As an aside, the observed strong variation in the methanol abundance in ices is not well understood theoretically. The formation of more complex organic species on grain surfaces is, however, limited by the accretion of atomic $\mathrm{C}$ (c.f., Fig. 3) and hence is set by the release of $\mathrm{C}$ from $\mathrm{CO}$ through cosmic-ray-produced UV photons in the gas phase (c.f., § 3.1) and only traces of complex species (complexorganics/CO $10^{-3}$ ) are expected in ice mantles through these processes. Unfortunately, absorption spectroscopy does not allow direct detection of trace species in interstellar ices and this important aspect of grain surface chemistry can only be tested indirectly through e.g., studies of the composition of hot cores where ices have been released into the gas phase (c.f., § 3.3).

\subsection{The chemistry of hot cores}

Hot cores are regions of warm $(>100 \mathrm{~K})$, dense $\left(>10^{6} \mathrm{~cm}^{-3}\right)$ gas near massive protostars that are characterized by the presence of high abundances of hydrogenated species, in particular $\mathrm{H}_{2} \mathrm{O}, \mathrm{NH}_{3}, \mathrm{H}_{2} \mathrm{CO}$, and $\mathrm{CH}_{3} \mathrm{OH}$ (Blake et al. 1987; Wright et al. 1996). In recent years, regions with similar characteristics as hot cores - but much smaller and therefore 
labeled hot corino's - have also been discovered around solar-type protostars (Bottinelli et al. 2004). Because of the similarity to the composition of interstellar ices, it is now generally accepted that these species reflect the sublimation of interstellar ices by the newly formed star when the dust reaches a temperature of $\sim 100 \mathrm{~K}$. The observed high abundance of deuterated species (e.g., HDCO, $\mathrm{D}_{2} \mathrm{CO}, \mathrm{CH}_{2} \mathrm{DOH}, \mathrm{CHD}_{2} \mathrm{OH}, \mathrm{CD}_{3} \mathrm{OH}$, $\mathrm{CH}_{3} \mathrm{OD}$, and $\mathrm{NH}_{2} \mathrm{D}$; Parise et al. (2006); Ceccarelli et al. (2001)) is also taken as evidence for the importance of ice sublimation in these regions as high deuteration is characteristic for regions of $\sim 10 \mathrm{~K}$, e.g., much colder than hot cores. In this view, ices are formed at low temperatures during the prestellar core phase of the molecular cloud and, after the formation of the protostar, the sublimated gas has not yet had the time to "equilibrate" with the gas (e.g., $\tau_{\text {chem }} \sim 10^{5} \mathrm{yr}$ ). Sputtering in shocks may also be important in releasing these molecules into the gas phase.

Hot cores also contain a multitude of more complex species such as ethyl alcohol, formic acid, dimethyl ether, methyl formate, methyl cyanide, and ethyl cyanide. The origin of these species is less obvious. Initially, like $\mathrm{H}_{2} \mathrm{O}$ and $\mathrm{CH}_{3} \mathrm{OH}$, they were thought to represent grain-surface products and putative chemical routes can be identified for species such as ethanol, dimethyl ether, and methyl formate (c.f., Fig. 3; Tielens \& Hagen (1982); Charnley \& Rodgers (2009)). However, the absence of quantitative reaction rates and branching ratios hampers detailed model efforts. At the same time, the nearimpossibility of detecting trace abundances of these species in interstellar ices severly limit observational approaches to assessing the importance of grain surface chemistry routes. In general, though, it can be expected that these species will have very low abundances given the low accretion rate of atomic C. Moreover, the presence of the nitrogen bearing species is difficult to understand in this scenario. As a result, a number of different chemical routes towards molecular complexity have been proposed. These are discussed in the next three subsections.

\subsubsection{Chemistry driven by evaporating ices}

The injection of $\mathrm{H}_{2} \mathrm{O}, \mathrm{CH}_{3} \mathrm{OH}$, and $\mathrm{NH}_{3}$ from the ices into the gas phase can drive a rich chemistry in these warm dense surroundings. Charnley et al. (1992) realized that methanol may play a key role in driving molecular complexity because protonated methanol will transfer $\mathrm{CH}_{4}^{+}$groups (rather than $\mathrm{H}^{+}$). Chemical routes towards the complex (oxide) species observed in hot cores can then be formulated. For example, the following route is available for dimethyl ether,

$$
\mathrm{H}_{3}^{+} \stackrel{\mathrm{H}_{2} \mathrm{O}}{\longrightarrow} \mathrm{H}_{3} \mathrm{O}^{+} \stackrel{\mathrm{CH}_{3} \mathrm{OH}}{\longrightarrow} \mathrm{CH}_{3} \mathrm{OH}_{2}^{+} \stackrel{\mathrm{CH}_{3} \mathrm{OH}}{\longrightarrow} \mathrm{CH}_{3} \mathrm{OCH}_{4}^{+} \stackrel{e}{\longrightarrow} \mathrm{CH}_{3} \mathrm{OCH}_{3}
$$

For hot cores rich in N-bearing species, $\mathrm{NH}_{3}$ is the precursor ice molecule which is channeled to $\mathrm{HCN}$ and then to $\mathrm{CN}$ in the gas. The reaction of cyanogen with specific hydrocarbons (also presumed to come from ices) leads then to the suite of N-bearing species observed (Charnley et al. 1992; Caselli et al. 1993). The timescale for the formation of these complex organics is some $3 \times 10^{4} \mathrm{yr}$ and after some $10^{5} \mathrm{yr}$ gas phase chemistry will have driven all the sublimated organics to CO.

Recent experiments have called these chemical routes into question. First, formation of some of the key intermediaries is inhibited ( Horn et al. 2004). Second, dissociative electron recombination leads to fragmentation (Geppert et al. 20006). In addition, the inferred chemical clock is difficult to reconcile with the rapid dynamical timescales associated with hot corinos. 


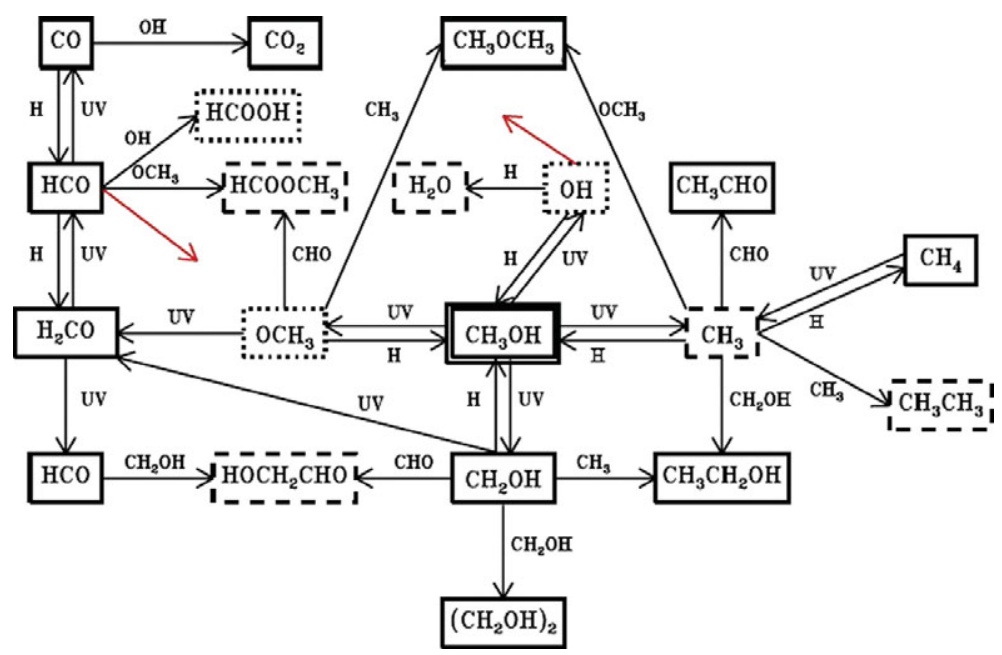

Figure 4. UV photolysis of ices containing $\mathrm{H}_{2} \mathrm{O}$ and $\mathrm{CH}_{3} \mathrm{OH}$ produces radicls which can react on to form more complex organic molecules. This figure illustrates a number of relevant chemical routes identified based upon laboratory experiments. Figure taken from Öberg et al. (2009).

\subsubsection{Energetic processing of interstellar ices}

UV photolysis and/or ion bombardment of interstellar ices will produce (hot) radicals - specifically $\mathrm{H} \& \mathrm{OH}$ - and some of these will react with other molecules while others will rattle around until they lose their excess energy and become trapped in the matrix. Upon warm up, the trapped radicals will diffuse and can recombine with each other, forming more complex species (Fig. 4). Recent laboratory studies have focused on putting these ideas on a firm footing identifying the chemical routes involved (Öberg et al. 2009; Hudson et al. 2008). Astronomical models have been developed, incorporating ice photochemistry under astrophysically relevant conditions (Garrod et al. 2008). These ideas are well worth pursuing further as a number of critical issues are examined. First, this photolysis is unlikely to be very chemical specific. Rather, starting with realistic interstellar ice mixtures, a large number of radicals can be produced which can recombine to a wide variety of species and isomers. It is unclear whether this chemical diversity can be reconciled with the astronomical record which points towards chemical specificity. Second, prolonged exposure to UV or particle radiation will lead to the production of macromolecular structures. Observationally, there is no evidence for such "organic goop" in the interstellar dust record. Also, this removes carbon from the complex organic inventory that is the focus of this review. The presence of $\mathrm{NH}_{3}$ and $\mathrm{H}_{2} \mathrm{CO}$ in the ices may provide an efficient polymerization route for the conversion of molecules to solid materials during warm up (Schutte et al. 1993) and this complication has to be evaluated. Finally, HCO is a key, initial product of the photolysis of ices containin CO but the telltale absorption signature of this molecule has never been observed, questioning the relevance of these chemical routes.

\subsubsection{Ions and interstellar ices}

Chemical schemes based upon $\mathrm{CH}_{3}$ transfer in ice-loaded environments - as originally proposed by Charnley et al. (1992) - are very elegant and appealing but the experimental evidence argues against it. However, a variant of this may be of relevance for the composition of hot cores. Specifically, it has become abundantly clear in recent years that 


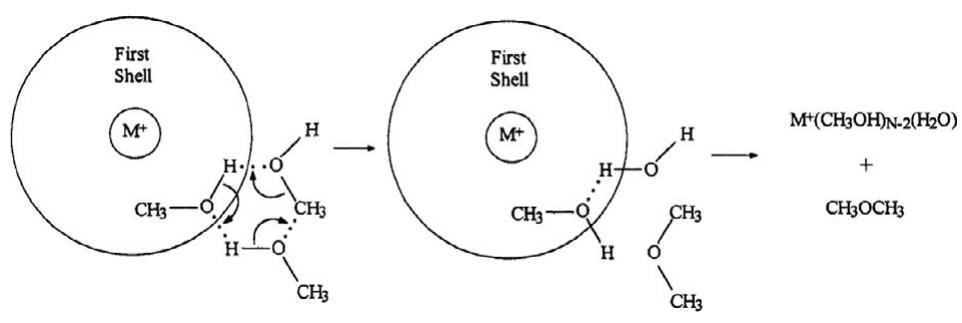

Figure 5. Possible chemical route towards molecular complexity in methanol-rich interstellar ices. The circle around the central (alkali) $\mathrm{M}^{+}$ion represents the first solvation shell of methanol. Methanol molecules in the second solvation shell and hydrogen bonded to the first are (stereochemically) in position to react. In this way, two methanol species are converted into dimethyl ether and water $\left(\mathrm{M}^{+}\left(\mathrm{CH}_{3} \mathrm{OH}\right)_{N} \rightarrow \mathrm{M}^{+}\left(\mathrm{CH}_{3} \mathrm{OH}\right)_{N-2} \mathrm{H}_{2} \mathrm{O}+\mathrm{CH}_{3} \mathrm{OCH}_{3}\right)$. Figure taken from Selegue \& Lisy (1994).

charge can be an important aspect for interstellar ices. First, the $4.62 \mu \mathrm{m}$ band is now generally attributed to the $\mathrm{OCN}^{-}$ion (Demyk et al. 1998). Second, any PAHs trapped in these ices can act as electron acceptors, localizing the charge and preventing electron diffusion and recombination (Bouwman et al. 2011). Likewise, trapped atoms such as $\mathrm{Na}$ and $\mathrm{K}$ can be efficient electron donors in interstellar ices. Third, recent studies on dipole alignment of molecular films (Balog et al. 2009) also point towards the importance of electric fields for the evolution of interstellar ices. Accepting that interstellar ices are charged and that these charges are localized, new interesting chemical routes akin to ion-molecule gas phase reactions but now in the solid state open up. Specifically, figure 5 illustates a possible route towards dimethyl ether, based upon molecular beams experiments on methanol droplets (Selegue \& Lisy 1994). So, the scenario would be: typical interstellar ices containing $\mathrm{CH}_{3} \mathrm{OH}$ and other species (eg., $\mathrm{H}_{2} \mathrm{O}$ ) including traces of alkali metals are charged by visible and UV photons. Upon warm up by a nearby protostar to $\sim 80 \mathrm{~K}$, the methanol segregates out from the water - known to be important in some interstellar sightlines (Boogert et al. 2000; Dartois et al. 1999) - and sterochemistry can ensue. The resulting more-weakly-bound dimethyl ether molecule can then readily sublimate. While there is good experimental support for this reaction scheme in droplets, the importance of methyl transfer without fragmentation in ices (on astrophysical timescales) is unknown and this whole scheme is highly speculative. Regardless, this discussion points towards issues that we have ignored in the past and that may actually be very important: charging of ices, stereochemistry, segregation, molecular solubility in water/methanol, and solid-state ion-molecule chemistry.

\section{The PAH reservoir}

Large ( 50 - 100 C-atom) PAH molecules are known to be abundant and ubiquitous in the interstellar medium. These molecules lock up $\sim 5 \%$ of the elemental $\mathrm{C}$ and hence represent a carbon reservoir which is comparable to that of gaseous CO. PAH species are often thought to form in the ejecta from C-rich AGB stars as critical intermediaries or byproducts of the dust formation process and detailed chemical routes based upon terrestrial soot studies have been proposed (Frenklach \& Feigelson 1989; Cherchneff et al. 1992). Alternatively, PAHs may result from shattering of carbonaceous materials in graingrain collisions in shocks in the interstellar medium itself (Jones et al. 1996). In the ISM, these molecular species will be further processed by UV and X-ray photons and 


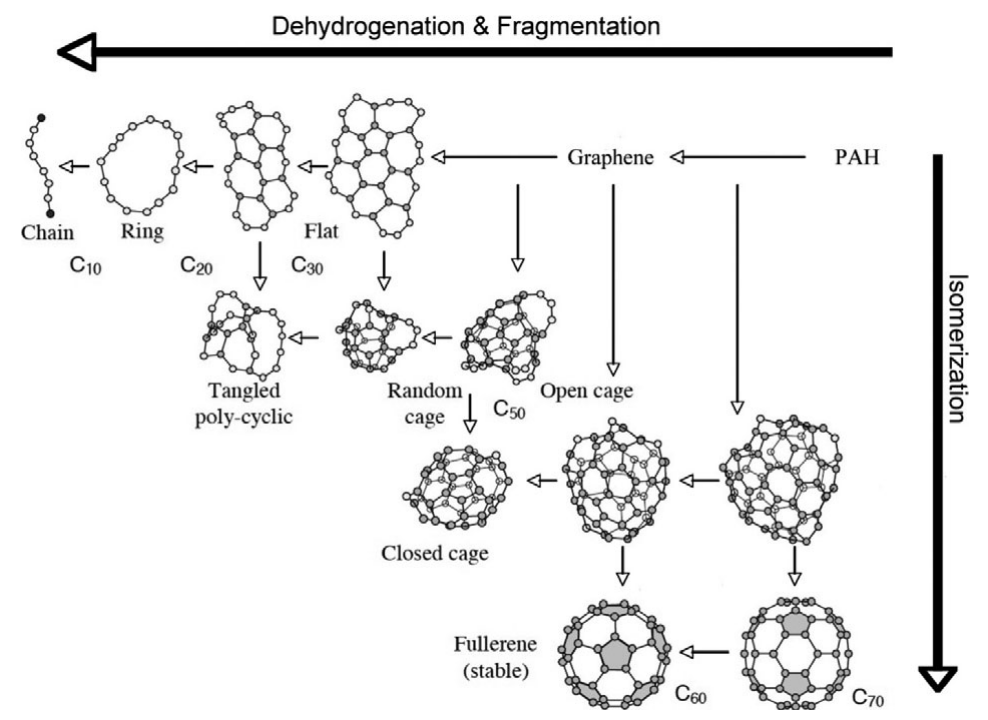

Figure 6. Chemical evolution of PAHs in the interstellar medium under the influence of UV photons combines the effects of dehydrogenation and fragmentation with those of isomerization. Fully hydrogenated PAHs - injected by stars into the ISM (top right side) - are photolyzed near bright stars, leading to complete H-loss (e.g., the weakest link) and the formation of graphene. Further fragmentation may lead to the formation of flats, rings, and chains. However, this process competes with isomerization to various types of stable intermediaries such as cages and fullerenes. Figure taken from Berné \& Tielens (2011).

by energetic particles in shock waves, hot gas or cosmic rays (Micelotta et al. 2010a,b, 2011), before becoming incorporated into regions of star and planet formation.

\subsection{PAH processing in the ISM}

Figure 6 schematically illustrates the chemical evolution of PAHs in space. Upon excitation, several fragmentation channels will compete with each other (and with IR fluorescence). Experiments show that loss of $\mathrm{H}$ dominates (Jochims et al. 1994; Joblin 2003). For typical interstellar conditions and relevant PAH sizes, there is then a kinetic equilibrium between fragmentation and $\mathrm{H}$-addition reactions. However, close to a star, $\mathrm{H}$-loss will dominate and PAHs are quickly stripped of all their H's leading to the formation of graphene structures (Le Page et al. 2001; Berné \& Tielens 2011). These can then fragment futher by losing C-atoms, forming rings, chains and small hydrocarbon radicals (Fig. 6). This fragmentation will compete with isomerization of graphene to cages and fullerene structures. The latter are much more stable to fragmentation than PAHs and can survive very close to a star. Given the calculated rapid destruction timescale of interstellar PAHs, it seems unavoidable that chemical growth of PAHs has to take place in the ISM. At large distances from stars, PAHs are in kinetic equilibrium with the H-flux and phenyl-like intermediaries can be abundant (Le Page et al. 2001). Laboratory studies show that such radicals can react with hydrocarbon species even at low tempertures and it is conceivable that this chemical growth process drives PAHs to ever larger sizes (Parker et al. 2011). The small hydrocarbon radicals "released" in the fragmentation may also set off a rich and interesting chemistry that may well contribute to the rich inventory of hydrocarbon radicals in photodissociation regions (Pety et al. 2005). Finally, while the focus here has been on fragmentation and chemistry driven by UV photon, similar fragmentation channels are available when PAHs are excited by energetic ions in 
shocks or hot plasmas (Postma et al. 2010). The details of these chemical routes have not yet been explored and dedicated experimental programs will be required to assess their viability (cf., Joblin (2003)).

\subsection{PAH processing in protostellar disks}

The photochemical PAH processing outlined in $\S 4.1$ is also very relevant for protoplanetary disks. Stellar UV photons may process PAHs in disk photospheres while X-ray photons from low mass $\mathrm{T}$ Tauri stars may penetrate deeply into the disk. X-rays will leave PAHs much more highly excited than the (non-ionizing) UV photons available in PDRs and hence may be particularly efficient in driving this type of photochemistry. PAHs can also be chemically processed by e.g., $\mathrm{H}$ and $\mathrm{OH}$ radicals in warm gas in the inner disk and early accretion phases (Kress et al. 2010). Figure 7 shows the calculated chemical timescale for PAH loss as a function of the temperature of the gas. The adopted pressure is typical for models of protoplanetary disks but the results are not very sensitive to this. PAHs are stable when the dynamical timescale of the system is shorter than this chemical timescale and typically this is the case for temperatures less than $1000 \mathrm{~K}$ (Kress et al. 2010). As this figure indicates, PAHs will be quickly destroyed $\left(<10^{6} \mathrm{yr}\right)$ in the inner regions $(<5 \mathrm{AU})$ of protoplanetary disks. The processes that created the chondrules in the Solar system likely also drove an interesting chemistry characterized by higher temperatures but shorter timescales. Finally, we note that the gas in the photosphere of the inner disk is also very warm but the PAHs themselves are not (except immediately after UV photon absorption). Hence whether this radical chemistry can play a role in the disk photosphere will need to be assessed.

Small hydrocarbon released by the destruction of PAHs (e.g., $\mathrm{C}_{2} \mathrm{H}_{2}$ ) will be partly oxidized to $\mathrm{CO}$ (and $\mathrm{CO}_{2}$ ) and partly channeled to $\mathrm{CH}_{4}$ and this is an effective way to drive the $\mathrm{CH}_{4} / \mathrm{CO}$ ratio to values more appropriate of "chemical equilibrium" at the temperatures and pressures of the disks. Small, catacondensed PAHs are more susceptible to attack by radicals than larger, pericondensed PAHs, resulting in faster chemical timescales for the former as compared to the latter. Temperatures and pressures are very conducive to chemical growth of PAHs as well and a fraction of the $\mathrm{C}_{2} \mathrm{H}_{2}$ released by the radical attack on catacondensed PAHs will flow along these routes. However, eventually, even the more stable PAHs will typically be destroyed by the radicals. Finally, we mention that if the diffusion timescale in the disk is much faster than the oxidation timescale, the hydrocarbons produced this way $\left(\mathrm{CH}_{4}, \mathrm{C}_{2} \mathrm{H}_{2}\right.$, and their radicals) will spread throughout the disk and be available for a rich organic chemistry (Gail 2002).

\section{Implications}

As emphasized in this review and illustrated in figure 1, the organic reservoir of the habitable zone of protoplanetary disks reflects a variety of chemical processes that release $\mathrm{C}$ from the otherwise unreactive reservoirs, $\mathrm{CO}$ and PAHs. For CO, hydrogenation on grain surfaces is key to activating the carbon. Subsequent ion-molecule chemistry either in the gas phase or in the solid state combined with warm up or photolysis \& warm up may then form a wide array of complex organics. For PAHs, photolysis can lead to fullerenes, cages \& hydrocarbon radicals, while warm radical chemistry destroys PAHs, forming methane, acetylene \& stable PAHs (temporarily), and (after diffusion) acetylenic derivatives. All of these processes likely played a role in setting the organic inventory of the Solar system and may have contributed to life's chemical start on the Earth. 


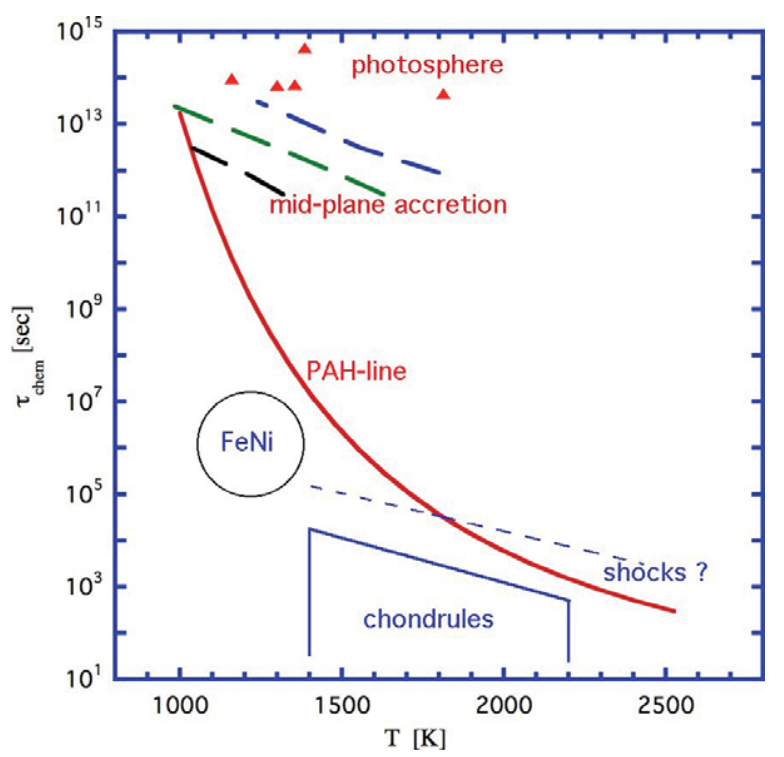

Figure 7. Chemical timescale for the breakdown of PAHs through chemical attack by $\mathrm{H}$ and $\mathrm{OH}$ radicals as a function of the temperature of the gas (Kress et al. 2010). At a given temperature, PAHs will be stable for timescales below the line marked "PAH-line". The black, green, and blue dashed lines indicate calculated temperatures in the mid plane at 1,2, and 5 AU (between $10^{4}-10^{6}$ yr) for a typical model for the evolution of the early Solar nebula (Bell et al. 1997). The box labeled chondrules gives the temperature-time regime experienced by chondrules in meteorites as derived from their texture (Connolly et al. 2006). The line labeled shocks is an indication that the processes producing chondrules may have lead to more extreme conditions without leaving traces in the meteorites. The circle indicates typical conditions derived from analysis of meteoritic FeNi (Meibom et al. 2000). The red triangles mark the temperatures derived from K-band CO observations (Najita et al. 2003) of a few T-Tauri stars for which accurate isochrone ages have been determined (Bertout et al. 2007).

\subsection{Chemical inventory of protoplanetary disks}

Infrared studies have revealed an interesting array of very abundant molecules in the warm gas of protoplanetary disks and hot cores including $\mathrm{CH}_{4}, \mathrm{H}_{2} \mathrm{O}, \mathrm{C}_{2} \mathrm{H}_{2}, \mathrm{HCN}$, and HNCO (Najita et al. 2003; Lahuis et al. 2007; Knez et al. 2009). Some of these are readily made in the warm dense gas while others are possibly ice-release-products. However, $\mathrm{C}_{2} \mathrm{H}_{2}$ and $\mathrm{HCN}$ are not typical surface chemistry products and the observed abundances in these regions are much higher than in prestellar cores, so they are not freeze out products either. Acetylene might be produced by the breakdown of PAHs $(\S 4.2)$ in the inner disk followed by rapid diffusion throughout the disk (Kress et al. 2010). Hydrogen cyanide also reflects a high temperature chemistry and may be produced in the same regions and perhaps even through the same processes if PAHs do contain $\mathrm{N}$ (Hudgins et al. 2005). The observed high abundances of species such as $\mathrm{C}_{2} \mathrm{H}_{6}$ in comets (Mumma et al. 2003) may reflect diffusion of PAH-breakdown-products (hydrocarbon radicals) and their reactions throughout the disk.

\subsection{Chemical inventory of comets}

Comets are generally thought to contain a pristine record of the prestellar core and/or the quiescent outskirts of the protosolar nebula. However, it is now clear that some components - crystalline silicates, CAI fragments - may well have been formed elsewhere in the Solar nebula and then diffused outwards. The chemical inventory of comets 


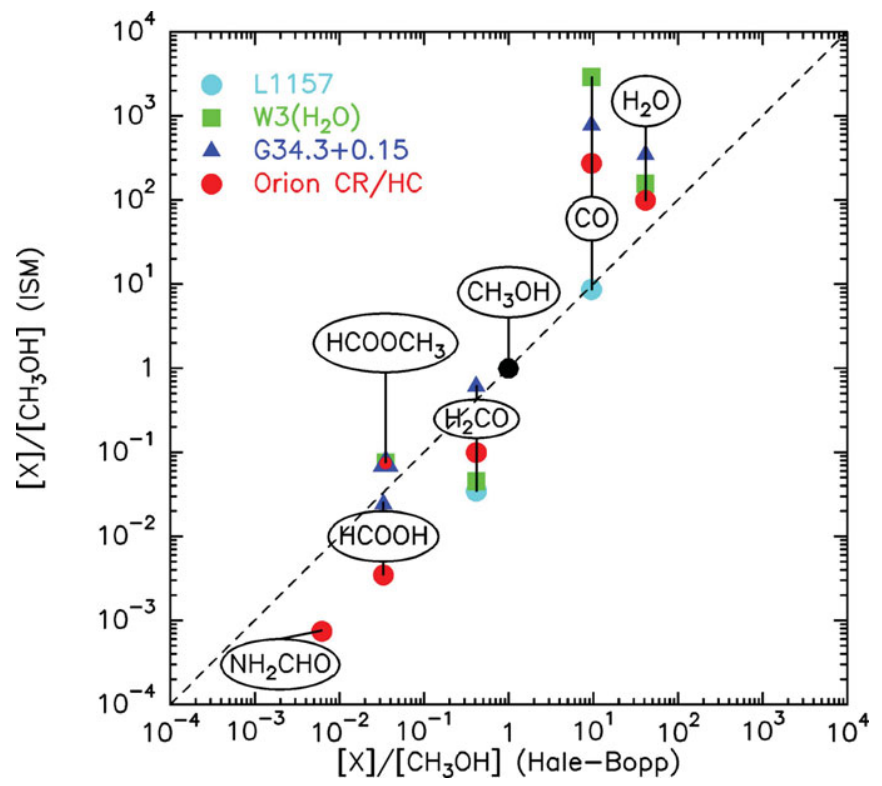

Figure 8. Comparison of the chemical composition of comets and hot cores. The commonality in chemical inventory and abundances of complex organic molecules points towards similarity in chemical processing involved. Figure taken from Bockelée-Morvan et al. (2000).

resembles that of hot cores with species such as methanol, formaldehyde, formic acid, methyl formate, and formamide. Even the observed abundances are quite similar (Fig. 8; Bockelée-Morvan et al. (2000). This resemblance of the organic inventory in composition and abundance may well point towards a shared chemical history. If cometary organics form by aggregating interstellar ice grains, then this would point towards an in situ origin for these complex organics - e.g., energetic processing of ices -, since more complex scenarios involving sublimation, gas phase reactions, macroscopic diffusion, and distillation would face difficulties preserving the relative abundances of these species.

\subsection{Chemical inventory of meteorites}

Carbonaceous meteorites are among the most primitive objects of the solar system. They exhibit significant carbon contents mostly occuring as insoluble organic matter. The macromolecular structure of this material resembles that of terrestrial kerogen $\dagger$. In addition, carbonaceous meteorites contain numerous soluble organic compounds. Many of these have familiar biochemical counterparts (e.g., amino acids, fatty acids, purines, pyrimidines, and sugars; Cronin \& Chang (1993); Botta \& Bada (2002) but the soluble organic inventory is very rich and includes alcohols, aldehydes, amides, amines, monoand dicarboxylic acids, aliphatic and aromatic hydrocarbons, heterocyclic aromatics, hydroxy acids, ketones, phosphonic and sulfonic acids, sulfides and ethers. Concentrations of the major representatives of these classes vary widely from less than 10 parts per million (amines) to tens of parts per million (amino acids) to hundreds of parts per million (carboxylic acids). The total soluble fraction can be as extreme as 0.3 (eg., Murchison) in CM carbonaceous meteorites.

In contrast to the Earth's biosphere, meteoritic biomolecules are a diverse and random mixture and this lack of specificity points towards an origin in abiotic processes. Both the soluble and the insoluble organic components of meteorites show an anomalous isotopic

$\dagger$ That should not be take to imply a biological origin for this material. 
composition in H, C, and N. In particular, the enrichment in D is evidence for formation at the low temperatures characteristics for the cold interiors of dense clouds rather than the warm nebular gas available in the asteroidal formation zone. Hence, it is generally accepted that the initial reservoir was inherited from the molecular cloud from which the Solar system formed and/or the outskirts of the Solar nebula. However, this record has largely been obliterated. In particular, the coexistence of soluble organics with clay minerals suggest active chemistry during the aquous (and thermal) alteration of the parent body, such as hydrolysis of nitriles and saturation of double bonds, and plausible reaction routes have been formulated (e.g., Strecker synthesis) (Peltzer \& Bada 1978). Similar processes likely played a role on the early Earth as well.

\section{Summary}

As outlined in this short review, the observed organic inventory of regions of planet formation as well as that of Solar system bodies is very rich and diverse, reflecting a wide variety of chemical processes contributing as material sojourns from their stellar "birth" sites to their planetary "resting" places. The two main molecular reservoirs of carbon in molecular clouds - $\mathrm{CO}$ and PAHs - are converted into a variety of more complex organic molecules through ion-molecule and grain surface chemistry in one case and UV photochemistry and radical chemistry in the other case (Fig. 1). Three different types of "scenarios" can be formulated differing in the intrinsic role of astrochemistry for the early emergence of life: 1) Delivery of carbon which is converted on the early Earth to the required building blocks (Urey-Miller hypothesis). 2) Delivery of a primed organic mixture $\left(\mathrm{H}_{2} \mathrm{CO}, \mathrm{HCN}\right)$ which were converted with the 'Strecker' mechanism into amino acids and the like on the Early Earth ("hamburger helper" model). 3) Delivery of the specific molecules necessary to jump start life ("Universal Darwinian pond"). On asteroids, process 2 seems to have take place. If asteroids delivered the organic inventory to Earth, then process 3 may well be key. Moreover, some laboratory studies show that processed interstellar ices - and therefore perhaps comets could as well - may well have delivered the essential ingredients which transformed into the first cells in water (Deamer et al. 2002). So, while the extent of astrochemistry's contribution to the origin of life is still to be discovered, it has become clear over the last decade that chemistry and biology are as important for understanding the Universe and our place in it as physics.

\section{References}

Balog, R., Cicman, P., Jones, N. C., \& Field, D., 2009, Phys Rev Let, 201, 73003

Bell, K. R., Cassen, P. M., Klahr, H. H., \& Henning, T. 1997, ApJ, 486, 372

Bergin, E. A., 2011, in The Interstellar Medium, ed: (Springer Verlag, Berlin), in press

Berné, O. \& Tielens, A. G. G.. M., 2011, submitted

Bertout, C., Siess, L., \& Cabrit, S. 2007, A \& A, 473, L21

Blake, G. A., Sutton, E. C., Masson, C. R., \& Phillips, T. G. 1987, ApJ, 315, 621

Bockelée-Morvan, D., et al. 2000, A $\&$ A, 353, 1101

Boogert, A. C. A., et al. 2000, A \& $A, 353,349$

Botta, O. \& Bada, J. L., 2002, Surveys in Geophysics, 23, 411

Bottinelli, S. \& et al., 2004, ApJ, 617, L69

Bouwman, J., Cuppen, H. M., Steglich, M., Allamandola, L. J., \& Linnartz, H. 2011, A $\mathscr{E}$ A, $529, \mathrm{~A} 46$

Caselli, P., Hasegawa, T. I., \& Herbst, E. 1993, ApJ, 408, 548

Ceccarelli, C., Loinard, L., Castets, A., Tielens, A. G. G. M., Caux, E., Lefloch, B., \& Vastel, C. $2001, A \& 3 A, 372,998$ 
Charnley, S. B., Tielens, A. G. G. M., \& Millar, T. J. 1992, ApJ, 399, L71

Charnley, S. B. \& Rodgers, S. B. 2009, Bioastronomy 200\%: Molecules, Microbes and Extraterrestrial Life, 420, 29

Cherchneff, I., Barker, J. R., \& Tielens, A. G. G. M. 1992, ApJ, 401, 269

Connolly, H. C., Desch, S. J., Ash, R. D., \& Jones, R. H., 2006, in Meteorites and the Early Solar System II (University of Arizona Press), p383

Cronin, J. R. \& Chang, S., 1993, in The Chemistry of Lifes Origin, eds. J.M. Greenberg, C.X. Mendoza-Gmez, and V. Pirronello, (Kluwer: Dordrecht), p209

Dartois, E., Demyk, K., d'Hendecourt, L., \& Ehrenfreund, P. 1999, A \& A, 351, 1066

Deamer, D., Dworkin, J. P., Sandford, S. A., Bernstein, M. P., \& Allamandola, L. J., 2002, Astrobiology, 2, 371

Demyk, K., Dartois, E., D'Hendecourt, L., Jourdain de Muizon, M., Heras, A. M., \& Breitfellner, M. $1998, A \& \mathcal{G} A, 339,553$

Frenklach, M. \& Feigelson, E. D. 1989, ApJ, 341, 372

Fuchs, G. W., Cuppen, H. M., Ioppolo, S., et al., 2009, A \& A, 505, 629

Gail, H.-P. 2002, $A$ \& $A$, 390, 253

Garrod, R. T., Weaver, S. L. W., \& Herbst, E. 2008, ApJ, 682, 283

Geppert, W., et al., 2006, Faraday discussions, 133, 177

Herbst, E. \& Klemperer, W. 1973, ApJ, 185, 505

Hidaka, H., Watanabe, N., Shiraki, T., et al., 2004, ApJ, 614, 1124

Hiraoka, K., Miyagoshi, T., Takayama, T., et al., 1998, ApJ, 498, 710

Hirota, T., Ohishi, M., \& Yamamoto, S. 2009, ApJ, 699, 585

Horn, A., et al., 2004, ApJ, 611, 605

Hudgins, D. M., Bauschlicher, C. W., Jr., \& Allamandola, L. J. 2005, ApJ, 632, 316

Hudson, R. L., Palumbo, M. E., Strazzulla, G., Moore, M. H., Cooper, J. F., \& Sturner, S. J., 2008, in The Solar System Beyond Neptune, eds: M. A. Barucci, H. Boehnhardt, D. P. Cruikshank, and A. Morbidelli, (University of Arizona Press, Tucson), p. 507

Ioppolo, S., Cuppen, H. M., Romanzin, C., et al., 2008, ApJ, 686, 1474

Joblin, C., 2003, SF2A-2003: Semaine de lAstrophysique Francaise, 175

Jochims, H. W., Ruhl, E., Baumgartel, H., Tobita, S., \& Leach, S. 1994,ApJ, 420, 307

Jones, A. P., Tielens, A. G. G.. M., \& Hollenbach, D. J., 1996, ApJ, 469, 740

Knez, C., Lacy, J. H., Evans, N. J., II, van Dishoeck, E. F., \& Richter, M. J. 2009, ApJ, 696, 471

Kress, M. E., Tielens, A. G. G. M., \& Frenklach, M. 2010, Advances in Space Research, 46, 44

Lahuis, F., et al. 2007, ApJ, 659, 296

Le Page, V., Snow, T. P., \& Bierbaum, V. M. 2001, ApJS, 132, 233

Meibom, A., Desch, S. J., Krot, A. N., Cuzzi, J. N., Petaev, M. I., Wilson, L., \& Keil, K. 2000, Science, 288, 839

Micelotta, E. R., Jones, A. P., \& Tielens, A. G. G. M. 2010, A \& A A, 510, A36

Micelotta, E. R., Jones, A. P., \& Tielens, A. G. G. M. 2010, $A \& \&$ A, 510, A37

Micelotta, E. R., Jones, A. P., \& Tielens, A. G. G. M. 2010, $A$ \& $A, 526,52$

Mumma, M. J., Disanti, M. A., dello Russo, N., Magee-Sauer, K., Gibb, E., \& Novak, R. 2003, Advances in Space Research, 31, 2563

Najita, J., Carr, J. S., \& Mathieu, R. D. 2003, ApJ, 589, 931

Öberg, K. I., Garrod, R. T., van Dishoeck, E. F., \& Linnartz, H. 2009, A \& A, 504, 891

Parise, B., Ceccarelli, C., Tielens, A. G. G. M., Castets, A., Caux, E., Lefloch, B., \& Maret, S. 2006, $A$ \& $A, 453,949$

Perker, D., et al., 2011, submitted

Peltzer, E. T. \& Bada, J. L., 1978, Nature, 272, 443

Pety, J., Teyssier, D., Fossé, D., Gerin, M., Roueff, E., Abergel, A., Habart, E., \& Cernicharo, J. 2005, $A$ \& $A, 435,885$

Postma, J., Bari, S., Hoekstra, R., Tielens, A. G. G.. M., \& Schlathter, T., 2010, ApJ, 708, 435

Requena-Torres, M. A., Martín-Pintado, J., Martín, S., \& Morris, M. R. 2008, ApJ, 672, 352

Schutte, W. A., Allamandola, L. J., \& Sandford, S. A. 1993, Icarus, 104, 118

Selegue, T. J. \& List, J. M., 1994, J Am Chem Soc, 116, 4874 
Tielens, A. G. G. M. \& Hagen, W. 1982, A \& A, 114, 245

Tielens, A. G. G. M. \& Allamandola, L. J., 2011, Physics and Chemistry at Low Temperatures, ed. L. Khriachtchev, (Pan Stanford, Singapore), p341

Wakelam, V., et al., 2010, Space Sci Rev, 156, 13

Watson, W. D. 1973, ApJ, 183, L17

Wright, M. C. H., Plambeck, R. L., \& Wilner, D. J. 1996, ApJ, 469, 216 